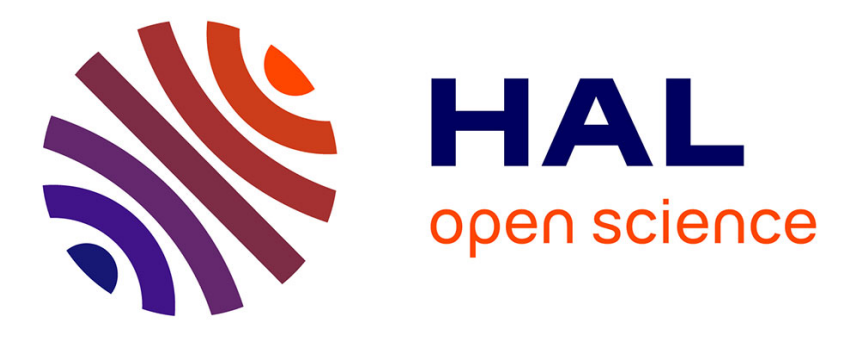

\title{
Spatial quantum noise of semiconductor lasers
}

Jean-Pierre Hermier, Alberto Bramati, A. Z. Khoury, Elisabeth Giacobino, Jean-Philippe Poizat, Tiejun Chang, Philippe Grangier

\section{To cite this version:}

Jean-Pierre Hermier, Alberto Bramati, A. Z. Khoury, Elisabeth Giacobino, Jean-Philippe Poizat, et al.. Spatial quantum noise of semiconductor lasers. Journal of the Optical Society of America. A Optics, Image Science, and Vision, 1999, 16 (11), pp.2140-2146. 10.1364/JOSAB.16.002140 . hal00558800

\section{HAL Id: hal-00558800 \\ https://hal-iogs.archives-ouvertes.fr/hal-00558800}

Submitted on 3 Apr 2012

HAL is a multi-disciplinary open access archive for the deposit and dissemination of scientific research documents, whether they are published or not. The documents may come from teaching and research institutions in France or abroad, or from public or private research centers.
L'archive ouverte pluridisciplinaire HAL, est destinée au dépôt et à la diffusion de documents scientifiques de niveau recherche, publiés ou non, émanant des établissements d'enseignement et de recherche français ou étrangers, des laboratoires publics ou privés. 


\title{
Spatial quantum noise of semiconductor lasers
}

\author{
J.-P. Hermier, A. Bramati, A. Z. Khoury, and E. Giacobino \\ Laboratoire Kastler Brossel, Université Pierre et Marie Curie, Ecole Normale Supérieure, \\ Centre National de la Recherche Scientifique, 4 place Jussieu, F-75252 Paris Cedex 05, France \\ J.-Ph. Poizat, T. J. Chang, and Ph. Grangier \\ Laboratoire Charles Fabry de l'Institut d'Optique, B.P. 147, F91403 Orsay Cedex, France
}

Received April 2, 1999; revised manuscript received May 26, 1999

\begin{abstract}
The transverse distribution of intensity noise in the far field of semiconductor lasers has been experimentally studied. For a single-mode edge-emitting laser, it has been found that a large amount of noise is present in higher-order nonlasing transverse modes parallel to the diode junction. In the case of a spatially multimode vertical-cavity surface-emitting laser, each mode exhibits a large noise, but these noises show strong anticorrelations. (c) 1999 Optical Society of America [S0740-3224(99)00909-1]

OCIS codes: $140.5960,140.2020,250.7260,270.6570,230.5440$.
\end{abstract}

\section{INTRODUCTION}

An understanding of the noise of semiconductor lasers has great importance in fields spanning from optical telecommunications to high-sensitivity spectroscopy. Intensity noise reduction bel ow the shot-noise level (SNL) was demonstrated first by Machida et al. ${ }^{1}$ and has been demonstrated since then by many others. ${ }^{2-4}$ In principle, the pumping mechanism of semiconductor lasers allows a faithful conversion of the sub-Poissonian electron statistics of the driving current to the photon statistics of the outgoing light. 5,6 This simple picture does not, however, work for all laser diodes, and many lasers exhibit larger noise. Possible reasons for this nonideal behavior are currently under investigation. For example, the importance of multimode effects ${ }^{7}$ on noise behavior has been demonstrated in the classical regime ${ }^{8-10}$ and recently in the quantum regime for longitudinal ${ }^{2-4,11,12}$ and polarization ${ }^{13}$ modes. In these experiments various modes were lasing, and the main effect was an anticorrelation among them because of mode competition. Such anticorrelations have also been observed between adjacent transverse modes with orthogonal polarizations in vertical-cavity surface-emitting lasers (VCSEL's). ${ }^{14-17}$

In this study we investigate the influence of transverse modes on the intensity noise of semiconductor lasers. Understanding the dynamics of higher-order transverse modes is of great importance when optimizing the coupling of a laser diode into an optical fiber or when diode pumping a solid-state laser. ${ }^{18}$ Moreover, investigating noise properties of a laser is a very sensitive way to obtain information about its dynamics. The appearance of noise in a mode can often be interpreted as a precursor for mode switching phenomena.

We have studied two different types of laser, namely, an edge-emitting laser, ${ }^{19}$ and a VCSEL. ${ }^{17}$ We have analyzed the spatial distribution of noise within the transverse profile of the laser beam by measuring its intensity noise after screening part of it in the far field with a mov- able razorblade (see Fig. 1). The fitting of the noise profile allows us to precisely identify the noisy spatial modes and to infer the presence of correlations between transverse modes. The present paper develops and compares the results originally presented in Refs. 17 and 19.

It will be shown below that the spatial noise behavior of edge-emitting lasers and of VCSEL's differs. For the VCSEL, the different transverse modes involved are above threshold and have no mutual coherence, owing to their different frequencies and polarizations. In contrast, the results presented for an edge-emitting laser describe a situation in which only the main mode is lasing and in which the transverse noise profile is due to an interference effect between this lasing mode and subthreshold higher-order transverse modes.

In Section 2 we introduce a simple model for describing the spatial dependence of quantum noise for both lasers under consideration. Then we present the experimental results obtained for the VCSEL (Section 3) and for the stripe laser (Section 4). Finally, the results obtained for the noise dependencies with the driving current in both lasers are presented and compared in Section 5.

\section{MODEL}

In this section we present the model describing the transverse distribution of quantum noise within the laser beam in the two cases corresponding to the VCSEL and to the edge-emitting laser diode. It is sufficient to consider only the lowest two transverse modes (labeled 0 and 1$),{ }^{20}$ with amplitudes given by

$$
\begin{aligned}
& u_{0}(x)=(2 / \pi)^{1 / 4} \sqrt{1 / w} \exp \left(-x^{2} / w^{2}\right), \\
& u_{1}(x)=(2 / \pi)^{1 / 4} \sqrt{1 / w} 2(x / w) \exp \left(-x^{2} / w^{2}\right),
\end{aligned}
$$

where $w$ is the size of the beam. The principle of the present experiments is to cut a part of the beam by means of a movable razorblade. Denoting by $X$ the position of 


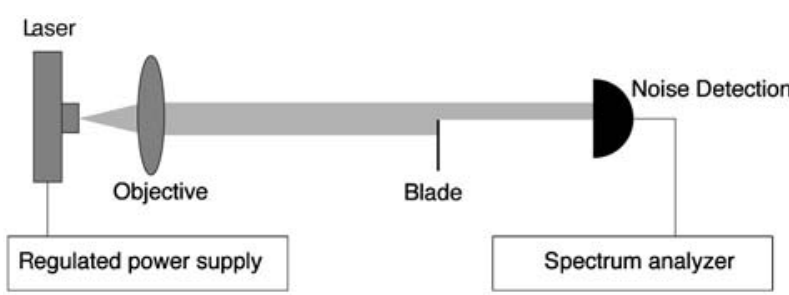

Fig. 1. Experimental setup for the measurement of the spatial intensity noise distribution.

the razorblade with respect to the beam center, we can conveniently define the quantities

$$
\begin{aligned}
& T_{0}(X)=\int_{X}^{\infty} u_{0}^{2}(x) d x, \quad T_{1}(X)=\int_{X}^{\infty} u_{1}^{2}(x) d x, \\
& Q_{01}(X)=\int_{X}^{\infty} u_{0}(x) u_{1}(x) d x .
\end{aligned}
$$

It is clear that $T_{0}(X)$ and $T_{1}(X)$ correspond to the fraction of the intensity of each mode after the blade, while $\mathrm{Q}_{01}(\mathrm{X})$ is the truncated overlap between the two modes. Owing to the normalization and the orthogonality of the mode functions, one obviously has $T_{0}(-\infty)=T_{1}(-\infty)$ $=1$ and $\mathrm{Q}_{01}(-\infty)=0$.

To calculate the quantum noise of the detected beam, one can choose from among various possible orderings of the field operators. Symmetrical ordering is often preferred to deal with squeezing effects; however, this requires that one carefully take into account all the vacuum modes that enter as a result of optical losses in the system. When chopping off the beam, this approach is not very convenient, and it is much simpler to use normal ordering. In the latter case the normally ordered variances are obtained from a calculation that is quite similar to the classical one. Then one obtains the true quantum noise by adding the shot noise (proportional to the mean intensity) to the result obtained through the normal ordering. ${ }^{21}$ As will be shown again below, squeezing effects are then recovered as negative values of the normally ordered variances. This approach is very convenient in the present context, and it will be used systematically below.

\section{A. Vertical-Cavity Surface-Emitting Laser}

We now consider the situation in which the two transverse modes are lasing. In the laser that is used, these two modes are orthogonally polarized, and their frequencies are separated by $\sim 100 \mathrm{GHz}$. We will thus incoherently add their intensities and neglect any beatnote between the two modes. From the definitions given above, the photon number $\langle n(X)\rangle$ in the chopped beam can be obtained simply as a function of the photon numbers $\left\langle\mathrm{n}_{0}\right\rangle$ and $\left\langle n_{1}\right\rangle$ in the TEM $M_{00}$ and TEM $M_{01}$ modes when the blade edge is at position $\mathrm{X}$ :

$$
\langle\mathrm{n}(\mathrm{X})\rangle=\mathrm{T}_{0}(\mathrm{X})\left\langle\mathrm{n}_{0}\right\rangle+\mathrm{T}_{1}(\mathrm{X})\left\langle\mathrm{n}_{1}\right\rangle .
$$

U sing standard methods, ${ }^{21}$ we can obtain the fluctuations in the beam as

$$
\begin{aligned}
\left\langle\delta \mathrm{n}^{2}(\mathrm{X})\right\rangle= & \left\langle\mathrm{n}^{2}(\mathrm{X})\right\rangle-\langle\mathrm{n}(\mathrm{X})\rangle^{2}=\mathrm{T}_{0}(\mathrm{X})\left\langle\mathrm{n}_{0}\right\rangle+\mathrm{T}_{1}(\mathrm{X}) \\
& \times\left\langle\mathrm{n}_{1}\right\rangle+\mathrm{T}_{0}{ }^{2}(\mathrm{X})\left\langle: \delta \mathrm{n}_{0}{ }^{2}\right\rangle+\mathrm{T}_{1}{ }^{2}(\mathrm{X})\left\langle: \delta \mathrm{n}_{1}{ }^{2}:\right\rangle \\
& +2 \mathrm{~T}_{0}(\mathrm{X}) \mathrm{T}_{1}(\mathrm{X})\left\langle\delta \mathrm{n}_{0} \delta \mathrm{n}_{1}\right\rangle
\end{aligned}
$$

where : : means that the operators are normally ordered. Let us define $C$, the degree of correlation between the two modes:

$$
\mathrm{C}=\frac{\left\langle\delta \mathrm{n}_{0} \delta \mathrm{n}_{1}\right\rangle}{\left(\left\langle\delta \mathrm{n}_{0}^{2}\right\rangle\left\langle\delta \mathrm{n}_{1}^{2}\right\rangle\right)^{1 / 2}} .
$$

In the case of perfect correlations, $\mathrm{C}$ is equal to 1 , whereas, in the case of perfect anticorrelations, $\mathrm{C}$ is equal to -1 . Using this notation, we find that

$$
\begin{aligned}
\left\langle\delta \mathrm{n}^{2}(\mathrm{X})\right\rangle= & \mathrm{T}_{0}(\mathrm{X})\left\langle\mathrm{n}_{0}\right\rangle+\mathrm{T}_{1}(\mathrm{X})\left\langle\mathrm{n}_{1}\right\rangle+\mathrm{T}_{0}{ }^{2}(\mathrm{X})\left\langle: \delta \mathrm{n}_{0}{ }^{2}:\right\rangle \\
& +\mathrm{T}_{1}{ }^{2}(\mathrm{X})\left\langle: \delta \mathrm{n}_{1}{ }^{2}:\right\rangle+2 \mathrm{~T}_{0}(\mathrm{X}) \mathrm{T}_{1}(\mathrm{X}) \\
& \times \mathrm{C}\left(\left\langle\delta \mathrm{n}_{0}{ }^{2}\right\rangle\left\langle\delta \mathrm{n}_{1}{ }^{2}\right\rangle\right)^{1 / 2} .
\end{aligned}
$$

We define the normalized variances as $v_{i}$ $=\left\langle: \delta \mathrm{n}_{\mathrm{i}}^{2}:\right\rangle /\left\langle\mathrm{n}_{\mathrm{i}}\right\rangle$. The value of $\mathrm{v}_{\mathrm{i}}$ represents excess noise if positive and squeezing if negative. The ratio between the intensities of the two modes $q$ is defined as $q$ $=\left\langle\mathrm{n}_{0}\right\rangle /\left\langle\mathrm{n}_{1}\right\rangle$. All the parameters used in this model have a physical significance and can be measured independently. The total intensity noise $S(X)$ when the razorblade is at position $\mathrm{X}$ and normalized to the shot noise of the full beam is thus

$$
\begin{aligned}
\mathrm{S}(\mathrm{X})= & \frac{1}{\mathrm{q}+1}\left\{\mathrm{qT}_{0}(\mathrm{X})+\mathrm{T}_{1}(\mathrm{X})\right. \\
& +\mathrm{T}_{0}^{2}(\mathrm{X}) \mathrm{qv}_{0}+\mathrm{T}_{1}^{2}(\mathrm{X}) \mathrm{v}_{1} \\
& \left.+2 \mathrm{~T}_{0}(\mathrm{X}) \mathrm{T}_{1}(\mathrm{X}) \mathrm{C}\left[\mathrm{q}\left(1+\mathrm{v}_{0}\right)\left(1+\mathrm{v}_{1}\right)\right]^{1 / 2}\right\} .
\end{aligned}
$$

We note that $S(X)$ is normalized, so the $S N L$ at position $X$ varies with the beam intensity and is therefore given by

$$
\mathrm{S}_{\mathrm{SNL}}(\mathrm{X})=\frac{\mathrm{qT}_{0}(\mathrm{X})+\mathrm{T}_{1}(\mathrm{X})}{\mathrm{q}+1} \text {. }
$$

\section{B. Edge-Emitting Laser}

For this laser, the experimental situation described in this subsection deals with a single lasing mode (mode 0 ). M ode 1 is bel ow threshold and therefore has a broad spectrum with a width that is larger than the free spectral range of the laser cavity. Although the central frequencies of modes 0 and 1 are different, there is a frequency overlap that allows interference between them. In a linearized approximation, the positive part $E^{(+)}(x)$ of the electric-field operator at position $\mathrm{x}$ will be given by

$$
\mathrm{E}^{(+)}(\mathrm{x})=\mathrm{E}_{0} \mathrm{u}_{0}(\mathrm{x})+\delta \mathrm{E}_{0}^{(+)} \mathrm{u}_{0}(\mathrm{x})+\delta \mathrm{E}_{1}^{(+)} \mathrm{u}_{1}(\mathrm{x}),
$$

where $E_{0}$ is the mean field of mode 0 , taken to be real, and $\delta \mathrm{E}_{0,1}^{(+)}$are the fluctuation operators of modes 0 and 1 . Owing to the spectrum overlap, the amplitude of mode 1 has to be coherently added to the main field amplitude. To first order, the intensity operator is thus

$$
\mathrm{n}(\mathrm{X})=\mathrm{T}_{0}(\mathrm{X}) \mathrm{E}_{0}^{2}+\mathrm{E}_{0}\left[\mathrm{~T}_{0}(\mathrm{X}) \delta \mathrm{P}_{0}+\mathrm{Q}_{01}(\mathrm{X}) \delta \mathrm{P}_{1}\right],
$$


where $\delta \mathrm{P}_{0,1}=\delta \mathrm{E}_{0,1}^{(+)}+\delta \mathrm{E}_{0,1}^{(-)}$are quadrature amplitude operators in phase with the mean field $E_{0}$. The quantum noise when the razorblade is at position $X$ is then given by

$$
\begin{aligned}
\left\langle\delta \mathrm{n}^{2}(\mathrm{X})\right\rangle= & \mathrm{T}_{0}(\mathrm{X}) \mathrm{E}_{0}^{2}+\mathrm{E}_{0}{ }^{2}\left[\mathrm{~T}_{0}{ }^{2}(\mathrm{X})\left\langle: \delta \mathrm{P}_{0}{ }^{2}:\right\rangle+\mathrm{Q}_{01}{ }^{2}(\mathrm{X})\right. \\
& \left.\times\left\langle: \delta \mathrm{P}_{1}^{2}:\right\rangle+2 \mathrm{~T}_{0}(\mathrm{X}) \mathrm{Q}_{01}(\mathrm{X})\left\langle\delta \mathrm{P}_{0} \delta \mathrm{P}_{1}\right\rangle\right] \\
= & \mathrm{T}_{0}(\mathrm{X})\left\langle\mathrm{n}_{0}\right\rangle+\mathrm{T}_{0}^{2}(\mathrm{X})\left\langle: \delta \mathrm{n}_{0}{ }^{2}:\right\rangle+\mathrm{E}_{0}{ }^{2}\left[\mathrm{Q}_{01}{ }^{2}(\mathrm{X})\right. \\
& \left.\times\left\langle: \delta \mathrm{P}_{1}^{2}:\right\rangle+2 \mathrm{~T}_{0}(\mathrm{X}) \mathrm{Q}_{01}(\mathrm{X})\left\langle\delta \mathrm{P}_{0} \delta \mathrm{P}_{1}\right\rangle\right]
\end{aligned}
$$

where the meaning of the various terms is as for $\mathrm{Eq}$. (5). The extra terms involving the overlap function $\mathrm{Q}_{01}(\mathrm{X})$ are clearly due to the fact that the main lasing mode acts here as a local oscillator that homodynes the noise of the higher-order modes. ${ }^{22}$

In this case the normalized variances are defined by $v_{i}=\left\langle: \delta \mathrm{P}_{\mathrm{i}}{ }^{2}:\right\rangle$, and the normalized correlation is given by

$$
\mathrm{C}=\frac{\left\langle\delta \mathrm{P}_{0} \delta \mathrm{P}_{1}\right\rangle}{\left[\left(1+\mathrm{v}_{0}\right)\left(1+\mathrm{v}_{1}\right)\right]^{1 / 2}}
$$

\section{EXPERIMENT WITH VERTICAL-CAVITY SURFACE-EMITTING LASERS}

\section{A. Preliminaries}

The VCSEL's used in these experiments are highquantum-efficiency oxide-confined GaAs/AIGaAs VCSEL'S with a diameter of $7 \mu \mathrm{m}$. They are made at the Department of Optoelectronics of the University of UIm, UIm, Germany. ${ }^{23}$ They consist of carbon-doped p-type AIGaAs/AIGaAs and silicon-doped n-type AIAs/AIGaAs Bragg reflectors with pairs of quarter-wavelength-thick layers. The top (bottom) mirror has a reflectivity of 99.8\% (99\%). The mirrors surround the three active 8-nm-thick GaAs quantum wells, the cladding layers, and the oxide aperture that provides both current and optical confinement. The devices are attached to a copper plate by use of silver paste and have an emission wavelength of $\sim 840 \mathrm{~nm}$. They have a very low threshold of $\mathrm{I}_{\text {tho }}$ $=1.2 \mathrm{~mA}$ and a fairly large quantum differential efficiency of $48 \%$. Above this driving current, the laser operates first in a single TE $\mathrm{M}_{00}$ mode up to the threshold of the first transverse mode, $I_{\text {th } 1}$. Both modes then lase together before a regime, $I>I_{\text {th2 }}$, is achieved, where the next transverse mode starts to oscillate. In Subsection 3. $B$ the region of interest is mainly between $I_{\text {th } 1}$ and $I_{\text {th2 }}$, when two and only two modes are lasing.

\section{B. Experimental Setup}

To take advantage of the principle of the pump noise suppression, a low-noise homemade power supply with an appropriate electronic filter provides the regulated el ectrical current that drives the VCSEL's. The device is also thermally stabilized with an active temperature stabilization. Thanks to this stabilization, we were able to operate the device at a fixed temperature with a drift as small as $0.01{ }^{\circ} \mathrm{C}$ per hour. The light beam is collimated by an antireflection-coated microscope objective located at a dis- tance of $2 \mathrm{~mm}$ from the laser output. The objective has a large numerical aperture (N.A., 0.6) to avoid optical losses, which would deteriorate the squeezing. The blade used to screen the beam is coated to avoid optical feedback into the laser. It is also mounted on a micrometric xy translation stage controlled by a motor. The motor enables us to gradually cut the beam over $50 \mathrm{~s}$.

To measure the intensity noise and the corresponding shot noise the standard scheme is to perform a balanced detection, by means of a pair of high-quantum-efficiency balanced photodiodes, on both sides of a beam splitter. The sum of the two photocurrents is proportional to the intensity noise, while the difference is proportional to the corresponding shot noise. ${ }^{24}$ Owing to the lasing of two modes with orthogonal linear polarizations, the shot noise obtained by a balanced detection would not be reliable. So only one photodiode is used (FND100; bandwidth, 10 kHz-30 MHz; quantum efficiency, 90\%). The SNL is obtained by balanced detection of a laser diode beam that has an intensity noise of $0.5 \mathrm{~dB}$ below the shot noise in the frequency range of $1-30 \mathrm{MHz}$. We carefully checked the linear dependence of the calibrated shot-noise signal with the optical power incident on the photodiodes. The shot noise obtained by this method was in agreement within $0.1 \mathrm{~dB}$ with the noise obtained by a thermal light generating the same dc current on the photodiode. The photodiode is connected via a low-noise homemade amplifier (with a National Semiconductor CLC425) and an electronic amplifier (Nucletude 4-40-1A) to a spectrum analyzer (Tektronix 2753P). With this setup the electronic noise was more than $6 \mathrm{~dB}$ below the signal that we measured for a typical detected power of $1.5 \mathrm{~mW}$. I $\mathrm{n}$ our experiment we could also perform a spectral analysis of the laser beam with a high-resolution monochromator $(0.03$ $\mathrm{nm}$ at 840-nm output). At the output of the monochromator a Glan polarizer (extinction ratio, $10^{-4}$ ) allows us to measure the polarization of the modes.

\section{Experimental Results}

The experimental procedure is as follows. To compare our experimental results with the theoretical predictions of the model presented in Section 2, we first need to measure the size of the beam [see Eq. (1)]. It is measured when the laser is single mode, that is, for a driving current $\mathrm{I}_{\text {op }}$ that is at an intermediate level between the threshold $I_{\text {tho }}$ of the TEM $M_{00}$ mode and the threshold $I_{\text {th } 1}$ of the TEM 01 mode. Note that the noise reduction levels given throughout this paper are corrected for the transmission of the optical components and the detectors' quantum efficiency. However, in the case of the measurement on VCSE L's, the losses of the microscope objective are not corrected for.

We then adjust the electrical driving current to have the VCSEL operating with only two transverse modes, i.e., $\mathrm{I}_{\text {th1 }}<\mathrm{I}_{\text {op }}<\mathrm{I}_{\text {th2 }}$. The two transverse modes are TEM $_{00}$ and TEM 01 (or TEM ${ }_{10}$ ). Using a monochromator and a polarizer, we can verify that TEM $\mathrm{T}_{00}$ (mode 0 ) and TEM $_{01}$ (mode 1 ) have linear polarizations orthogonal to each other. We also measure the intensity of each mode (separating them with a Glan polarizer), which gives the values of $\left\langle n_{0}\right\rangle$ and $\left\langle n_{1}\right\rangle$. From the measured intensity noise of each mode we determine the values of the excess 
noises $v_{0}$ and $v_{1}$. The correlation [Eq. (6)] can be determined by use of $v_{0}, v_{1}$, and the value of the total intensity noise $\left\langle\delta \mathrm{n}^{2}(-\infty)\right\rangle$.

Our model then provides the value of $\left\langle\delta \mathrm{n}^{2}(\mathrm{X})\right\rangle$ for every position of the blade. Finally, we measure the total intensity noise and the total intensity of the beam versus the position of the blade and compare it with the prediction of the model. Note that the theoretical trace is not obtained by an optimization procedure, since all the parameters are measured beforehand separately.

In Fig. 2(a) we have plotted the experimental results and the predictions of our model for the noise at $15 \mathrm{MHz}$ of a first VCSEL. The anticorrelations are equal to -0.75 for these curves. As expected, since $v_{0}$ and $v_{1}$ are large, we observe large variations of the intensity noise with the position of the blade. The agreement between theory and experiment is also very good. In Fig. 2(b) we have plotted the experimental results and the predictions of our model for a second VCSEL, which presented a higher anticorrelation parameter: $\quad C=-0.98$. The variations of the intensity noise are even larger. If we compare the results of Figs. 2(a) and 2(b), we also notice that the shape of the curves depends on the value of the correlations. The agreement between experimental re-

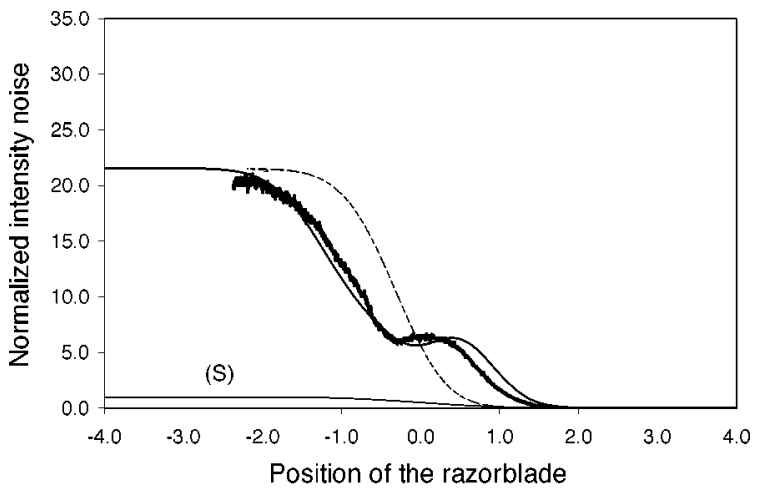

(a)

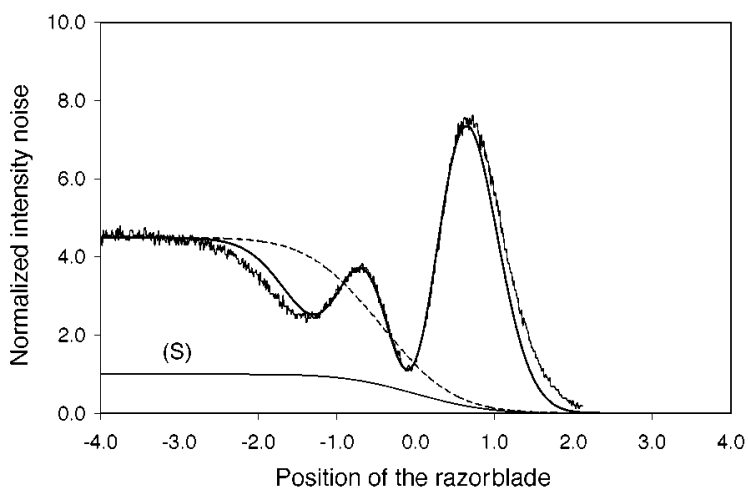

(b)

Fig. 2. Normalized intensity noise of a VCSEL versus the position of the razorblade, normalized to the beam size. The smooth (solid) trace is the theoretical prediction, and the noisy trace corresponds to the experimental results. Trace (S) is the SNL (proportional to the intensity). The dashed curve shows the theoretical intensity noise corresponding to an overall beam attenuation equal to the transmission of the razorblade. The fitting parameters are as follows: (a) For VCSEL 1: $C=-0.75, v_{0}$ $=25.82, v_{1}=330.9$, and $q=6.13 ;$ (b) for VCSEL 2: C $=-0.98, v_{0}=80.2, v_{1}=3648, q=34.8$. sults and theoretical predictions is again very good. These results confirm the validity of our hypothesis, particularly the fact that we have taken only two modes into account and that we have neglected the contribution of the nonlasing modes.

Note that the partial screening by the blade can reduce the noise by more than a simple attenuation effect. This is shown in Fig. 2, where for some ranges of the blade position the intensity noise goes clearly below the noise of the full beam attenuated down to the actual intensity of the cut beam. For various positions of the blade, the intensity noise can be considerably increased or decreased, depending on the contributions of the two modes and their correlation. Note also that, when the blade is exactly halfway through $(X=0)$, the intensity noise is equal to the intensity noise of the beam uniformly attenuated by $50 \%$. This result derives from the fact that intensity noise profiles of the different transverse modes are even functions.

\section{EXPERIMENT WITH EDGE-EMITTING LASERS}

\section{A. Experimental Setup}

The single-mode semiconductor laser that was used is a Fabry-Perot, multiple-quantum-well, index-guided AlGaAs device (SDL 5411-G1) emitting at $810 \mathrm{~nm}$. It has a single lasing transverse mode. It is collimated by a highN.A. (0.65) aspherical lens and is stabilized by the $24 \%$ feedback of an external grating. The zeroth order of the grating couples out $60 \%$ of the light power. The external cavity ensures truly single-longitudinal-mode operation. ${ }^{4}$ The threshold of the laser system is $I_{\text {th }}=17 \mathrm{~mA}$. The differential quantum efficiency measured at the output of the grating is $32 \%$. In this configuration its intensity noise is $16 \%$ below the SNL, and its longitudinal sidemode rejection rate is $>35 \mathrm{~dB} .4,25$ The values of the noises given bel ow correspond to the real noise at the output of the grating.

The experimental setup ${ }^{19}$ is similar to the one used for the VCSEL experiment (see Fig. 1). The intensity noise detection is a standard balanced scheme that allows a convenient comparison between the intensity noise and the SNL. ${ }^{24}$ The noise analysis frequency of the results presented bel ow is $14 \mathrm{MHz}$, but the frequency dependence of the effects is essentially flat within the bandwidth of our detectors $(2-20 \mathrm{MHz})$. Note that an edge-emitting laser does not present a cylindrical symmetry. One obtains all the results presented below by scanning the razorblade al ong the direction parallel to the junction plane. Owing to a different optical guiding, no interesting transverse effects take place in the direction perpendicular to the diode junction.

\section{B. Experimental Results}

The results for a laser-diode driving current of I $=100 \mathrm{~mA}$ are displayed in Fig. 3. The intensity profile presents the Gaussian shape of the TE $\mathrm{E}_{00}$ mode. The SN L that is proportional to the intensity therefore exhibits an error function shape as the blade cuts the beam. As can be seen in Fig. 3, the intensity noise has a very different shape. This difference signifies that the noise transverse distribution is different from the mean intensity distribu- 


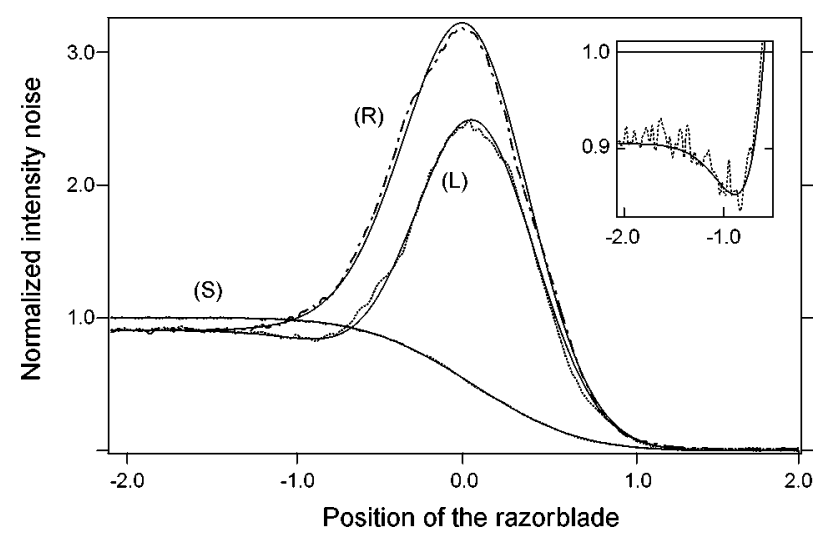

Fig. 3. Normalized intensity noise of an edge-emitting laser versus the position of the razorblade, normalized to the beam size. Trace (S) is the SNL (proportional to the intensity). Trace (L) $[(R)]$ corresponds to the razorblade entering the beam from the left (right). For each trace the solid curve is the best fit. Trace (R) has been flipped over for convenient comparison with trace $(\mathrm{L})$. The fitting parameters are $\mathrm{v}_{0}=-0.10 \pm 0.02, \mathrm{v}_{1}=14.9$ $\pm 0.2, v_{2}=1 \pm 0.5, C=0.23 \pm 0.02$. The inset, a zoom of the SNL-normalized noise of trace $(\mathrm{L})$, shows that the maximum squeezing is obtained when part of the beam is screened. For each trace, the solid curve is the best fit.

tion and that noise is present in higher-order spatial modes. Note that the noise maximum is around the $X$ $=0$ position of the blade. This corresponds to fluctuations in the beam position. To identify these modes, and to quantify their noise and correlations, the experimental scans were fitted with the simple theoretical model presented in Section 2.

When looking carefully at expression (12), which gives the intensity noise as a function of the blade position, one can observe that the correlation term changes sign, depending on whether the razorblade enters the beam from the right or from the left, owing to the following equality:

$$
\mathrm{Q}_{01}(\mathrm{X}) \equiv \int_{\mathrm{X}}^{+\infty} \mathrm{u}_{0}(\mathrm{x}) \mathrm{u}_{1}(\mathrm{x}) \mathrm{dx}=-\int_{-\infty}^{-\mathrm{X}} \mathrm{u}_{0}(\mathrm{x}) \mathrm{u}_{1}(\mathrm{x}) \mathrm{dx} \text {. }
$$

The correlation term therefore has a different sign, de pending on whether the noise is measured on the righthand or the left-hand half of the beam. This means that, when the correlation is nonzero, the noise level on the right-hand side of the beam is different from the noise level on the left-hand side. This is indeed what was observed experimentally (see Fig. 3).

In Fig. 3 we compare a scan taken with the blade entering the beam from the right $(R)$ and a scan taken with the blade entering from the left $(L)$. Their different shapes show that the correlations are nonzero. The maximum noise value is different for each scan, showing that the intensity noises of the two halves are different. This symmetry breaking is due either to a slight misalignment of the grating or to a small defect in the semiconductor laser. As for the VCSEL, these correlations lead to the a priori surprising result that one can improve the full beam squeezing by chopping off part of the beam, as is shown in the inset of Fig. 3.

As can be seen from Fig. 3, the experimental results are well fitted by the model. The fits are performed in the following way. The arithmetic average of traces $(L)$ and
(R) $\{$ i.e., $[(L)+(R)] / 2\}$ is first fitted with the correlation coefficient set to zero. One obtains $v_{0}=-0.10, v_{1}$ $=14.9$, and $v_{2}=4$. One then fits each trace by using the established values of these parameters to obtain the value of the correlation coefficients. One obtains $\mathrm{C}^{\mathrm{L}}$ $=-0.23 \pm 0.02$ and $C^{R}=0.22 \pm 0.02$ for traces $(L)$ and $(R)$, respectively, where one should have $C^{L}=-C^{R}$, according to $\mathrm{Eq}$. (12). The negative value for $\mathrm{v}_{0}$ indicates $10 \%$ squeezing.

Note that the fits include the noise power $v_{2}$ of the second-order $\mathrm{TE}_{20}$ mode, although the contribution of this mode was not given in the model, for clarity. All the correlations involving this $\mathrm{TE}_{20}$ mode have been set to zero. However, the fits are not very sensitive to the value of $v_{2}$, which explains that the uncertainty regarding its value is quite large.

\section{DEPENDENCY OF INTENSITY NOISES WITH THE DRIVING CURRENT}

In this section the behavior of the noise in the various modes is examined as a function of the laser driving current.

In Fig. 4(a) are displayed the intensity noises of modes 0 and 1 , and the total intensity noise of the VCSEL, for values of the current for which only these two modes are lasing. This corresponds to the situation discussed in Section 3. We also measured the noises of modes 0 and 1 , separating them with a Glan polarizer. As is qualitatively expected from an independent mode theory for above-threshold modes, ${ }^{6}$ their noises decrease with the

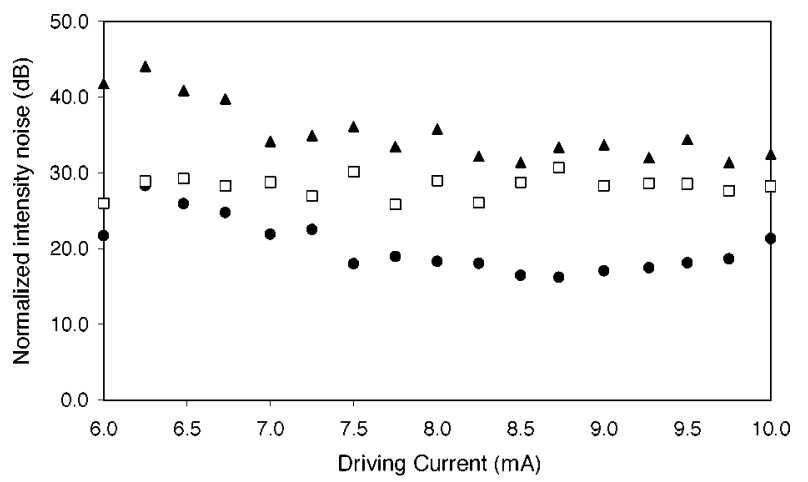

(a)

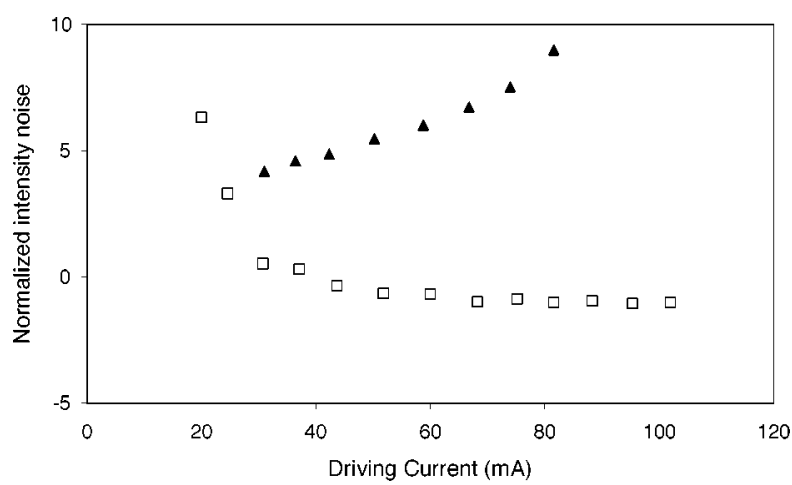

(b)

Fig. 4. Normalized intensity noise of the total beam (points), of mode 0 (squares), and of mode 1 (triangles) versus the driving current (a) for the VCSEL and (b) for the edge-emitting laser. 
driving current. The total intensity noise, which is measured by removal of the polarizer, is smaller than the noise of any of the two modes, owing to their anticorrelations.

The noise levels $v_{0}$ and $v_{1}$ of modes 0 and 1 , respectively, for the edge-emitting laser are plotted in Fig. 4(b) as a function of the driving current. Here $\mathrm{v}_{0}$ is measured directly from the noise of the total beam, whereas $v_{1}$ is inferred from the fit. It can be seen that the noise $v_{1}$ increases with the driving current. For a subthreshold mode, one can indeed expect the noise level to increase as the driving current increases and to diverge at threshold. According to the standard model for noise in laser diodes, ${ }^{6}$ this noise varies as $8 g^{2} /(g-\alpha)^{2}$, where $g$ is the laser gain and $\alpha$ the losses. Note that the noise $\mathrm{v}_{0}$ of mode 0 goes below the shot noise but remains higher than the theoretical value $1-\eta$, where $\eta$ is the overall quantum efficiency. The reason for this discrepancy is currently under investigation and might be linked to transversemode effects.

The observed behavior of mode 1 as a function of driving current is therefore consistent with our claim that this mode is above threshold in the VCSEL and below threshold for the edge-emitting laser.

\section{CONCLUSIONS}

We have analyzed in this paper the spatial distribution of the intensity of a VCSE L and of an edge-emitting laser, and we have shown that the transverse noise distribution can be very different from the transverse intensity distribution. We have found that these two types of semiconductor laser exhibit different behaviors. In a VCSEL the two transverse modes lase at different frequencies. The intensities of the two modes then add to each other incoherently, and the anticorrelation effects appear between the intensity fluctuations of both modes. In a singlemode edge-emitting laser the effect is coherent, and the observed correlations involve an interference of a subthreshold transverse mode with the main lasing $T E_{00}$ mode. A qualitative difference between the two lasers is that the phase-sensitive effect of the edge-emitting laser leads to a different intensity noise profile, depending on whether the blade enters the beam from the right or from the left.

An important result of this study is that, in both cases, one can reduce the intensity noise relative to the shot noise by partially screening the beam, owing to the noise correlations between the different transverse modes. It must be borne in mind that this effect is purely a spatial effect. The VCSEL presents intensity (anti)correlations stemming from mode competition that is due to the homogeneous part of the gain spectrum, as is reported in Refs. $2,4,7,13,15$, and 16 . In the case of the edge-emitting laser, the correlations are between field amplitudes, and their physical origin might be related to the so-called $\mathrm{Pe}$ termann excess noise factor ${ }^{26-28}$ and to loss-induced coupling. ${ }^{29}$

\section{ACKNOWLEDGMENTS}

We thank P. Schnitzer, R. Michalzik, and K. J . Ebeling (University of UIm, Germany) for providing us with the
VCSEL samples. This research was supported by the European Strategic Program for $R$ \& $D$ in Information Technology (Advanced Quantum Information Research 20029) and by the European Community Training Mobility Researchers Microlasers and Cavity Quantum Electrodynamics programs (contract ERBFMRX CT 96-00066). A. Bramati's work was supported by Training Mobility Researchers fellowship ERBFMBI CT 950204.

J.-P. Hermier can be reached at the address on the title page, by telephone at 33-1-4427-4393, or by e-mail at hermier@spectro.jussieu.fr.

\section{REFERENCES}

1. S. Machida, Y. Yamamoto, and Y. Itaya, "Observation of amplitude squeezing in a constant-current-driven semiconductor laser," Phys. Rev. Lett. 58, 1000-1003 (1987).

2. S. Inoue, H. Ohzu, S. Machida, and Y. Yamamoto, "Quantum correlation between longitudinal-mode intensities in a multimode squeezed semiconductor laser," Phys. Rev. A 46, 2757-2765 (1992).

3. H. Wang, M. J . Freeman, and D. G. Steel, "Squeezed light from injection-locked quantum well lasers," Phys. Rev. Lett. 71, 3951-3954 (1993).

4. F. Marin, A. Bramati, E. Giacobino, T.-C. Zhang, J .-Ph. Poizat, J.-F. Roch, and P. Grangier, "Squeezing and intermode correlations in laser diodes," Phys. Rev. Lett. 75, 4606-4609 (1995).

5. Yu. M. Golubev and I. V. Sokolov, "Photon antibunching in a coherent light source and suppression of the photorecording noise," Sov. Phys. J ETP 60, 234-238 (1984).

6. Y. Yamamoto, S. Machida, and O. Nilsson, "Amplitude squeezing in a pump-noise-suppressed laser oscillator," Phys. Rev. A 34, 4025-4042 (1986).

7. A. W. Smith and J. A. Armstrong, "Intensity noise in multimode GaAs Iaser emission," IBM Syst. J. 10, 225-232 (1966).

8. D. Pieroux and P. Mandel, "Transient dynamics of a multimode laser: oscillation frequencies and decay rates," Opt. Commun. 107, 245-248 (1994).

9. K. Otsuka, D. Pieroux, and P. Mandel, "Modulation dynamics in a multimode laser with feedback," Opt. Commun. 108, 273-277 (1994).

10. G. P. Agrawal and N. K. Dutta, Semi conductor Lasers (Van Nostrand Reinhold, New York, 1993).

11. C. Becher, E. Gehrig, and K.-J . Boller, "Spectrally asymmetric mode correlation and intensity noise in pump-noisesuppressed laser diodes," Phys. Rev. A 57, 3952-3960 (1998).

12. S. Lathi and Y. Yamamoto, "I nfluence of nonlinear gain and loss on the intensity noise of a multimode semiconductor laser," Phys. Rev. A 59, 819-825 (1999).

13. D. C. Kilper, D. G. Steel, R. Craig, and D. R. Scifres, "Polarization-dependent noise in a photon-number squeezed light generated by quantum-well lasers," Opt. Lett. 21, 1283-1285 (1996).

14. E. Goobar, J. W. Scott, B. Thibeault, G. Robinson, Y. Akulova, and L. A. Coldren, "Noise in homodyne and heterodyne detection," Appl. Phys. Lett. 67, 3697-3699 (1995).

15. D. C. Kilper, P. A. Roos, J. L. Carlsten, and K. L. Lear, "Squeezed light generated by a microcavity laser," Phys. Rev. A 55, R3323-R3326 (1997).

16. F. Marin and G. Giacomelli, "Polarization and transverse mode dynamics of VCSELs," Quantum Semiclassic. Opt. 1, 128-132 (1999).

17. A. Bramati, J.-P. Hermier, A. Z. Khoury, E. Giacobino, P. Schnitzer, R. Michalzik, K. J. Ebeling, J.-Ph. Poizat, and Ph. Grangier, "Spatial distribution of the intensity noise of a vertical-cavity surface-emitting laser," Opt. Lett. 24, 893895 (1999). 
18. C. C. Harb, T. C. Ralph, E. H. Huntington, D. E. McClelIand, H.-A. Bachor, and I. Freitag, "Intensity-noise dependence of $\mathrm{Nd}$ :YAG lasers on their diode-laser pump source," J. Opt. Soc. Am. B 14, 2936-2945 (1997).

19. J .-Ph. Poizat, T.-J . Chang, O. Ripoll, and Ph. Grangier, "Spatial quantum noise of laser diodes," J . Opt. Soc. Am. B 15, 1757-1761 (1998).

20. We assume that operation involves the first two transverse modes with Hermite-Gauss profiles, which corresponds to the experimental situation, both for VCSEL's and for edgeemitting lasers.

21. M. O. Scully and M. S. Zubairy, Quantum Optics (Cambridge U. Press, Cambridge, UK, 1997).

22. M. D. Levenson, W. H. Richardson, and S. H. Perlmutter, "Stochastic noise in TEM $M_{00}$ laser beam position," Opt. Lett. 14, 779-781 (1989); M. D. Levenson, S. H. Perlmutter, andW. H. Richardson, "Stochastic position noise, or why a laser beam cannot go straight," in Quantum Optics V, J. D. Harvey and D. F. Walls, eds. (Springer-Verlag, Heidel berg, 1989).

23. D. Wiedenmann, P. Schnitzer, C. J ung, M. Grabherr, R. J äger, R. Michalzik, and K. J . Ebeling, "Noise characteristics of $850 \mathrm{~nm}$ single-mode vertical cavity surface emitting lasers," Appl. Phys. Lett. 73, 717-719 (1998).
24. H. P. Yuen and V. W. S. Chan, "Noise in homodyne and heterodyne detection," Opt. Lett. 8, 177-179 (1983).

25. M. J. Freeman, H. Wang, D. G. Steel, R. Craig, and D. R. Scifres, "Wavelength-tunable amplitude-squeezed light from a room-temperature quantum-well laser," Opt. Lett. 18, 2141-2143 (1993).

26. K. Petermann, "Calculated spontaneous emission factor for double-heterostructure injection lasers with gain-induced waveguiding," IEEE J . Quantum Electron. QE-15, 566-570 (1979).

27. H. A. Haus and S. Kawakami, "On the "excess spontaneous emission factor' in gain-guided laser amplifiers," IEEE J . Quantum Electron. QE-21, 63-69 (1985).

28. A. E. Siegman, "Excess spontaneous emission in nonHermitian optical systems. I. Laser amplifiers," Phys. Rev. A 39, 1253-1263 (1989); "Excess spontaneous emission in non-Hermitian optical systems. I. Laser oscillators," 39, 1264-1268 (1989); see also Lasers (University Science, Mill Valley, Calif., 1986).

29. Ph. Grangier and J .-Ph. Poizat, "A simple quantum picture for the Petermann excess noise factor," Eur. Phys. J . D 1, 97-104 (1998); "Quantum derivation of the excess noise factor in lasers with nonorthogonal eigenmodes," Eur. Phys. J . D. (to be published). 